\title{
HIV-1 Penetrates Coronary Artery Endothelial Cells by Transcytosis
}

\author{
Chandrasekhar Gujuluva, ${ }^{1}$ Alan R. Burns, ${ }^{2}$ Tatiana Pushkarsky, ${ }^{3}$ Waldemar Popik, ${ }^{4}$ Omri Berger, ${ }^{1}$ \\ Michael Bukrinsky, ${ }^{3}$ Michael C. Graves, ${ }^{1}$ and Milan Fiala ${ }^{1}$ \\ ${ }^{1}$ Departments of Medicine, and Neurology, UCLA School of Medicine, Los Angeles, California, USA \\ ${ }^{2}$ Department of Medicine, Section of Cardiovascular Sciences and Leukocyte Biology, Baylor College of \\ Medicine, Houston, Texas, USA \\ ${ }^{3}$ The Picower Institute for Medical Research, Manhasset, New York, USA \\ ${ }^{4}$ Oncology Center, The Johns Hopkins University School of Medicine, Baltimore, Maryland, USA \\ Accepted November 13, 2000
}

\begin{abstract}
Background: The pathogenesis of HIV-1-related cardiomyopathy is poorly understood, but HIV-1 has been detected in cardiomyocytes. Whether HIV-1 penetrates into the myocardium by infection of coronary artery endothelial cells (CAEC) or using transcellular or paracellular routes across CAEC has not been resolved.

Materials and Methods: A model of the CAEC barrier was constructed with primary CAEC (derived from human coronary vessels). Polymerase chain reaction (PCR) assay, infectious assay, and immunofluorescence were employed to show abortive nature of HIV-1 infection of CAEC. Tight junction (TJ) and cell adhesion proteins were visualized by immunofluorescence. The time course of HIV-1 invasion was measured by HIV-1 RNA assay. Inulin permeability assay determined paracellular leakage. Transmission electron microscopy demonstrated virusinduced endothelial vacuolization.

Results: Despite a strong display on CAEC of CXCR4 and a lesser expression of CCR3 and CCR5, HIV-1 did not
\end{abstract}

productively replicate in CAEC, as shown by infectious assay, immunofluorescence, and electron microscopy. HIV-1 infection of CAEC was abortive with minimal reverse transcription of strong stop DNA and pol but not fulllength or two LTR DNA circles. Upon infection of the model with 1 million RNA copies of HIV- $1_{J R-F L}$, virus penetration $2 \mathrm{hr}$ postinfection (PI) was negligible but increased by $1750 \% 24 \mathrm{hr}$ PI. The paracellular permeability increased during this period by only $25 \%$. Neither AOP-RANTES nor v-MIPII significantly reduced HIV- $l_{J R-F L}$ invasion. Virus infection did not alter the integral $\mathrm{TJ}$ protein occludin and the TJ-associated protein ZO-1. HIV-1 exposed CAEC and brain microvascular endothelial cells (BMVEC) developed extensive cytoplasmic vacuolization with retroviral-like particles in the vacuoles.

Conclusions: The endothelium is not an impenetrable barrier to HIV-1. The virus opens a transcellular route across coronary and brain endothelia in cytoplasmic vacuoles.

\section{Introduction}

Even as advances in AIDS management have lead to improved survival from opportunistic infections, HIV-1 cardiomyopathy has become an increasingly common complication in AIDS patients. Postmortem cardiac abnormalities in patients with AIDS include most commonly pericardial effusion and myocarditis with dilated cardiomyopathy and less often endocarditis and coronary vasculopathy (1). A large prospective study of asymptomatic HIV-1positive patients revealed an annual incidence rate of dilated cardiomyopathy of 15.9 cases per 1000 patients. In the majority of the patients with

Address reprint requests to: Milan Fiala, MD, UCLA Neurology, 710 Westwood Plaza, Los Angeles, CA 90095-1769.

Phone: 310-206-6392; Fax: 310-794-5094; E-mail fiala@ucla.edu myocarditis, rare HIV-1 nucleic acids were detected in the cardiomyocytes. In addition, coinfection with other viruses, such as Coxsackie group B, cytomegalovirus, and Epstein-Bar virus may be synergistic with HIV-1 in the pathogenesis of myocardiopathy (2).

The mechanisms of HIV-1 entry into the myocardium have not been elucidated. Virus invasion could be initiated during the stage of intensive plasma viremia early after primary infection. We previously demonstrated a paracellular route of HIV-1 passage across brain endothelium, which is breached by inflammatory cytokines (3) or cocaine (4). As with some other viruses, HIV-1 could penetrate into cells by a transcellular route, via receptormediated endocytosis (5) through clathrin-coated pits (6) or through caveolae (7), and could exit by 
exocytosis on the abluminal side. HIV-1 could also increase endothelial permeability by induction of proinflammatory cytokines. For example, tumor necrosis factor- $\alpha$ (TNF- $\alpha$ ) is able to reorganize the actin cytoskeleton in glomerular epithelial cells (8) and endothelia $(9,10)$, and interleukin (IL) -6 can enhance endothelial permeability (11). Furthermore, vasoactive substances, in particular vascular permeability factor/vascular endothelial growth factor (VPF/VEGF), increase vascular permeability in certain pathologic and physiologic states by induction of vesiculo-vacuolar organelles (VVO), which are interconnected clusters of vesicles and vacuoles traversing venular endothelium (12). Human herpes virus 8 associated with Kaposi's sarcoma codes for proteins with homologies to chemokines and cytokines, including a viral form of IL-6 that binds directly to and activates gp 130 (13), a signaltransducing protein expressed by all cells.

Another possible mode for HIV-1 invasion of the heart is by the "Trojan" transport in lymphocytes and monocytes (14). Monocyte transmigration into the brain may be increased in patients abusing cocaine, because this abused drug was shown in vitro to enhance monocyte migration across a blood-brain barrier (BBB) model (15). Finally, HIV-1 could invade the brain and alter the barrier function by infecting endothelia and budding off the abluminal surface. That HIV-1 could infect brain microvascular endothelial cells (BMVEC) productively has been observed by one group (16), but not by another (17). In the former study, BMVEC released p24 antigen in the supernatant and displayed intracellular p24 antigen, whereas in the latter study HIV-1 replication was minimal and virus could be detected only by cocultivation with a T-cell line. With other types of endothelia, the results of HIV-1 infection have been heterogeneous: productive with liver and glomerular capillary endothelial cells and some human umbilical vein endothelial cell (HUVEC) strains (18) but nonproductive with other HUVEC strains (19).

In our previous work, we demonstrated that in cocaine abusing individuals HIV-1 might penetrate across brain endothelia by a paracellular route opened by TNF- $\alpha(3,4)$. Here we have investigated whether the virus could penetrate the endothelia by a transcellular route, a paracellular route, or by replication. HIV-1 did not propagate in coronary endothelia but a small fraction of the virus penetrated the endothelial barrier, apparently by transcytosis through cytoplasmic vacuoles induced by the virus.

\section{Materials and Methods \\ Cell Culture}

Coronary artery endothelial cells (CAEC) (Clonetics/ Bio Whittaker Inc., Walkersville, MD, USA) were isolated by the producer from normal human heart (large vessel) coronary arteries by enzymatic digestion, and were propagated in $75 \mathrm{~cm}^{2}$ TC flasks using $\mathrm{EGM}^{\mathrm{R}}-2-\mathrm{MV}$ Bullet Kit medium (Clonetics/ Bio Whittaker Inc). CAEC were received from the manufacturer in passage 2 . These cells were utilized up to passage 8 .

The CAEC model was constructed with CAEC monolayer on a polyethylene terephthalate (PET) membrane ( $3 \mu \mathrm{m}$ or $8 \mu \mathrm{m}$ pore size) in a Tissue Culture (TC) insert (Falcon Plastics, Beckton Dickinson, Franklin Lakes, NJ, USA). The PET membrane was coated with rat-tail collagen type I ( $5 \mu \mathrm{g} / \mathrm{insert})$ and human fibronectin ( $5 \mu \mathrm{g}$ /insert) according to manufacturer's instructions (both from Collaborative Biomedical Products, Bedford, MA, USA). To prepare the model, 100,000 CAEC in $0.3 \mathrm{ml}$ growth medium were seeded into the upper chamber of a TC insert and the insert was placed into a well of a 24-well plate containing $1.0 \mathrm{ml}$ medium. After 4 days, the monolayer became confluent, as evaluated by phase contrast microscopy, immunostaining with ZO-1 antibody, and electron microscopy.

CEM is a T-cell line susceptible to productive infection with HIV-1. 8E5/LAI is a CD4 ${ }^{+}$CEMderived T-cell line, each cell containing a single integrated copy of LAV (20). The BMVEC model of the BBB was prepared as described previously (3).

\section{Molecular and Viral Permeability of the CAEC Model}

Permeability assays were done with three replicate models for each treatment level. Molecular permeability was assessed by a diffusion assay, as described previously (3). Briefly, 100,000 CPM of ${ }^{14} \mathrm{C}$ carboxylated inulin (MW 5000, Sigma Chemical Co., St. Louis, MO, USA) was placed in the upper chamber and, after a 2-hr incubation, $50 \mu \mathrm{L}$ of each lower and upper chamber contents was removed, counted, and the permeability coefficient was determined.

Viral penetration studies were performed in replicate models using a challenge with 1 million RNA copies of HIV- $1_{\text {JR-FL }}$ in the upper chamber. After the indicated time interval, an aliquot of medium was obtained from the lower chamber and virus concentration was determined by the HIV RNA (Amplicor) assay, as described previously (3). The modified chemokine AOP-RANTES and the viral chemokine vMIP-II were obtained from A. Proudfoot, Serono Research Institute, Geneva, Switzerland, and were used at a dilution of $100 \mathrm{ng} / \mathrm{ml}$, as described (21).

\section{Antibodies}

Immunofluorescent characterization of CAEC was performed with the antibodies to factor VIII-related antigen (rabbit polyclonal), ZO-1 (mouse monoclonal), occludin (rabbit polyclonal), $\beta$-catenin (all from Zymed Laboratories, South San Francisco, CA, USA), PECAM-1 (mouse clone WM-59 from Pharmingen, San Diego, CA, USA), and ICAM-2 (rabbit polyclonal from RED, Minneapolis, MN, USA). 


\section{Virus and Viral Assays}

The HIV-1 strains included R5-tropic strain, HIV-1 $1_{\mathrm{JR}}$ FL (molecular clone provided by I. Chen, UCLA), and X4-tropic strains HIV-1 $1_{\text {LAI }}$ (obtained through the AIDS Research and Reference Reagent Program, NIAID, NIH, Bethesda, MD, USA), and HIV-1 $1_{\text {NL 4-3. }}$. The stock of HIV-1 $1_{\text {LAI }}$ (approximately $5 \times 10^{9}$ RNA copies $/ \mathrm{ml}$ ) was prepared in peripheral blood mononuclear cells stimulated with phytohemagglutinin A. The stock of HIV- $1_{\mathrm{NL4}-3}$, which was prepared by transfecting Jurkat CD4 ${ }^{+}$T cells with the DNA of the infectious molecular clone, was purified by ultracentrifugation through a cushion of sucrose buffer, as described previously (22). The titer of the purified preparation of HIV- $1_{\mathrm{NL} 4-3}$ was $1.5 \times 10^{9} \mathrm{CPM} / \mathrm{ml}$ measured by the reverse transcriptase (RT) activity assay (22), which corresponds to $3 \times 10^{8} \mathrm{pg} / \mathrm{ml}$ of $\mathrm{p} 24$ or $3 \times 10^{12}$ virions $/ \mathrm{ml}$.

Viral replication studies were performed in CAEC monolayers, which were grown in 6-well plates, by infection with HIV-1 $1_{\text {LAI }}$ virus at a multiplicity of 100 RNA copies/cell. Viral titers were determined at the indicated time intervals by Coulter HIV-1 p24 antigen assay kit (Beckman Coulter, Inc., Miami, FL, USA). The Coulter antigen assay is based on the use of a murine monoclonal antibody against p24 coated in a microtiter plate. The test was performed according to manufacturer's instructions. The p24 antigen standards (Coulter R HIV-1 p24 antigen kinetic standard, Miami, FL, USA) (0.0078-4.0 $\mathrm{ng} / \mathrm{ml}$ ) were run in parallel with the samples.

Cell lysates for DNA PCR studies were prepared from CAEC grown in 100-mm diameter dishes grown to $80 \%$ confluency. After washing the cell monolayer with phosphate-buffered saline/1\% bovine serum albumin (PBS/BSA), the virus was added to cells in $4 \mathrm{ml}$ PBS/BSA at different multiplicities for $2 \mathrm{hr}$ at $37^{\circ} \mathrm{C}$. At the end of infection, unadsorbed virus was removed and plates were washed three times with PBS/BSA, and growth medium was added and incubated until harvested by trypsinization and resuspension in a solution of trypsin neutralizing buffer. The cells were spun, frozen, and used for viral transcript analysis.

Immunofluorescence Thirty thousand endothelia were grown to confluence for 4 days in gelatincoated, glutaraldehyde cross-linked 16-well chamber slides (Nalge Nunc International, Naperville, IL, USA), as described previously (23). After fixation for 30 min with PBS containing 4\% paraformaldehyde at RT, the cells were permeabilized with $0.1 \%$ Triton X-100 for 10 min, blocked with $1 \%$ BSA, incubated for $1 \mathrm{hr}$ with a primary antibody (monoclonal or polyclonal antibody at $5 \mu \mathrm{g} / \mathrm{ml}$ ), washed, and then incubated with FITC-conjugated secondary antibody at the recommended concentration. For dual staining, two primary mouse and rabbit antibodies and two secondary FITC- or Texas Red-conjugated antibodies specific for mouse or rabbit IgG were used. The stained cells were viewed using an Olympus BH60 microscope with a mercury lamp attachment or a Zeiss LSM 310 confocal laser microscope.

Transmission Electron Microscopy CAEC or brain endothelial monolayers on coverslips were prepared as above and were infected with $\mathrm{HIV}-\mathrm{l}_{\mathrm{NL}-43}$ at a multiplicity of 500,000 or greater RNA copies per cell. After $24 \mathrm{hr}$, the cells were fixed with PBS containing $3 \%$ glutaraldehyde for $1 \mathrm{hr}$, and scraped with a razor blade into PBS. The cells were post-fixed with $1 \%$ tannic acid and $1 \%$ osmium tetroxide, followed by staining with uranyl acetate and lead citrate, and embedded in LX 112 resin (Polysciences, Inc., Warrington, PA, USA), as described previously (23). The sections were viewed using a JEOL 200CX transmission electron microscope.

\section{PCR for HIV-1 Reverse Transcription in CAEC}

CAEC $(400,000$ cells in a 6-well plate), were exposed to 1 million or higher number of RNA copies of HIV-1 $1_{J R-F L}$ or HIV-1 $1_{\text {LAI }}$ that had been pretreated with $400 \mathrm{U} / \mathrm{ml}$ RNase-free DNAse I (BoehringerMannheim, Indianapolis, IN, USA) in PBS containing $0.04 \mathrm{M} \mathrm{MgCl}_{2}$ (1 hr at room temperature). After $4 \mathrm{hr}$ of incubation with the virus in a minimum volume, the cells were washed four times with PBS and further cultivated in $\mathrm{EGM}^{\mathrm{R}}$-2-MV Bullet Kit medium. At indicated time intervals after infection, the cells were scraped, centrifuged, and the cell pellet was frozen. After thawing the cell pellets, they were lysed in $200 \mu \mathrm{l}$ PCR buffer, composed of $50 \mathrm{mM} \mathrm{KCl}, 10 \mathrm{mM}$ Tri-HCl (pH 8.3), $2.5 \mathrm{mM} \mathrm{MgCl} 2,0.1 \mathrm{mg}$ gelatin $/ \mathrm{ml}$, $0.45 \%$ Nonidet P-40, $0.45 \%$ Tween 20 , and $100 \mu \mathrm{g} / \mathrm{ml}$ proteinase $\mathrm{K} / \mathrm{ml}(24)$. After protein digestion $(2 \mathrm{hr}$ at $\left.56^{\circ} \mathrm{C}\right)$ and inactivation of the proteinase $(10 \mathrm{~min}$ at $\left.95^{\circ} \mathrm{C}\right), 25 \mu \mathrm{l}$ cell lysate was subjected to 25 cycles $(\alpha-$ tubulin primers) or 35 cycles (primers for LTR R/U5 \{(L) 5'-GGC TAA CTA GGG AAC CCA CTG-3', (R) 5'-CTG CTA GAG ATT TTC CAC ACT GAC-3'\} and pol \{(L) 5'-TTC TTC AGA GCA GAC CAG-3'(R) 5'ACT TTT GGG CCA TCC ATT-3'\} of amplification as described (24). To evaluate the sensitivity of each primer pair, different dilutions of the lysate prepared from 8E5/LAI cells (containing one proviral copy per cell) were amplified in parallel. Amplified DNA was analyzed by Southern blot hybridization with ${ }^{32} \mathrm{P}$ labeled probes (probe for R/U5 5'-TGTGTGCCCGTCTGTTGTGTG-3'; nucleotides 557-577; probe for pol 5'-GGAAGCTCTATTAGATACAGG-3').

\section{Results}

Immunochemical and Ultrastructural Characteristics of CAEC

Upon cell confluence, CAEC monolayer assumed cobblestone appearance. The endothelia displayed peri- 

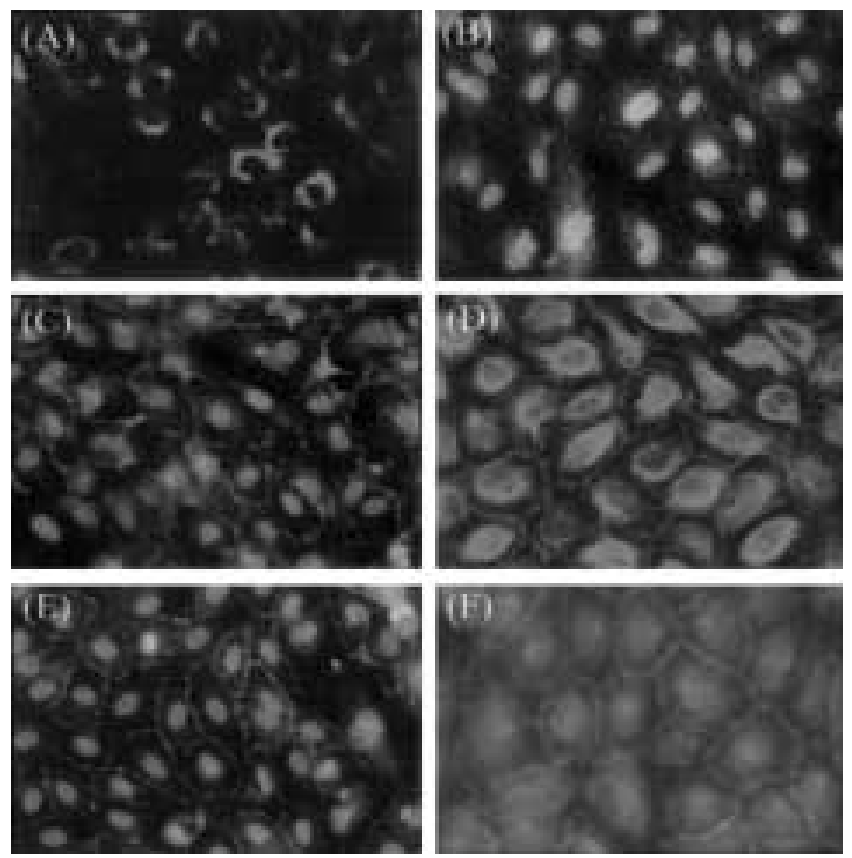

Fig. 1. Surface, tight junction, and adhesive proteins on CAEC. One hundred thousand CAECs were grown to confluence on glutaraldehyde-fixed gelatin-coated 16-well chamber slides for 5 days. The cells were fixed for 20 min with $4 \%$ paraformaldehyde at room temperature. The cells were stained using the immunofluorescence technique with the following antibodies: factor VIII-related antigen (A), ICAM-2 (B), PECAM-1 (C), $\beta$-catenin (D), ZO-1 (E), and occludin (F).

nuclear staining with antibody to factor VIII-related antigen and $\beta$-catenin, and rim staining with antibodies to the tight junction (TJ) transmembrane proteins occludin and claudin, the $\mathrm{TJ}$-associated peripheral membrane proteins $\mathrm{ZO}-1$ and $\mathrm{ZO}-2$, and the cell adhesive proteins PECAM-1 and ICAM-2 (Fig. 1). Control staining of CAEC with isotype-matched antibodies was negative, as reported previously (25). We showed previously that in vitro CAEC strongly display the chemokine receptor CXCR4, and less strongly CCR3 and CCR5. Coronary endothelia in the heart tissues are decorated with CXCR4, CCR5, and CCR3 (25).

Transmission electron microscopy of CAEC cultured on a porous membrane exhibited the characteristics of primary endothelia with a confluent monolayer of tightly adjoining and very thin cells. Rare tight junctions were noted at cell borders (Fig. 2).

HIV-1 Infection of CAEC Is Abortive Restricted to R/U5 and pol Reverse Transcription

The supernatant media of CAEC infected with HIV- $\mathrm{l}_{\text {LAI }}$ were tested by the p24 antigen assay at $5 \mathrm{hr}, \mathrm{l}$ day, and 7 days postinfection (PI) and all were negative for HIV-1 p24 antigen. CEM cells infected by identical HIV- $1_{\text {LAI }}$ inoculum produced a high amount of p24 antigen $(0.05 \mathrm{ng} / \mathrm{ml})$ in the supernatant. No p24 HIV-1 antigen was detected by

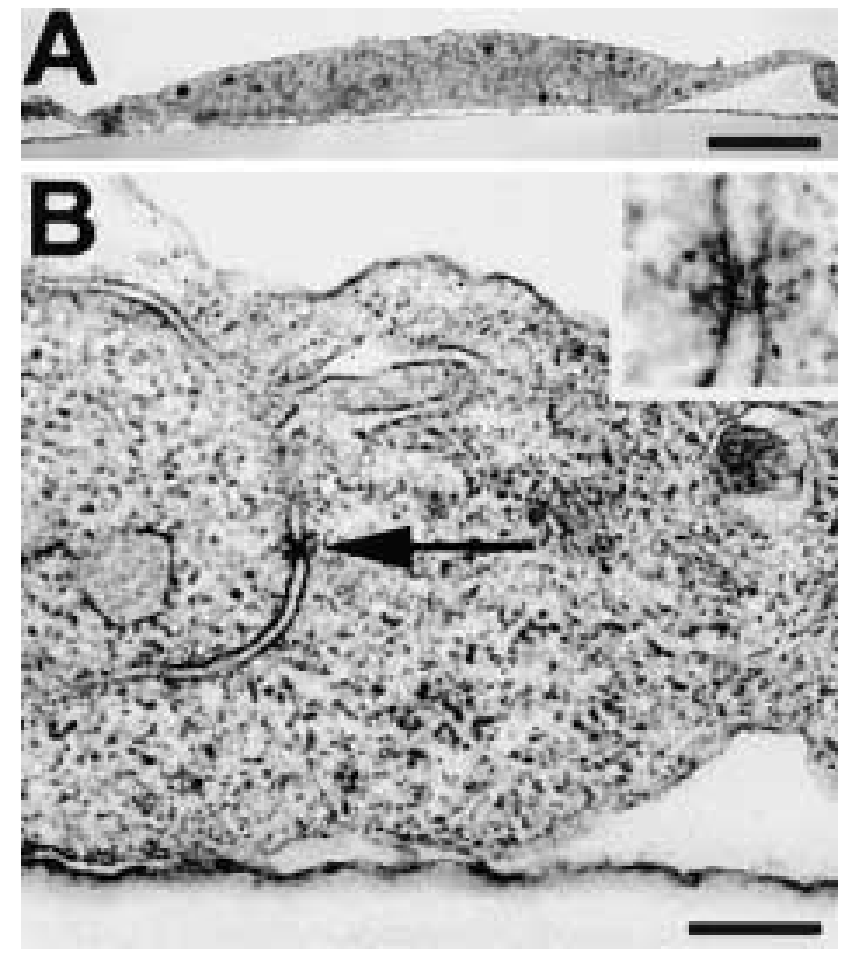

Fig. 2. Transmission electron microscopy of CAEC. A monolayer of CAEC displays tight junctions at each cell border contacting the adjacent cell. Note cell thinness and paucity of vacuoles. The monolayer was grown on a terepthalate membrane ( 3 micron pore size) in a cell culture insert (Falcon Plastics). (B) Tight junction between two CAEC.

immunofluorescence 5 days PI in CAEC or BMVEC infected by either the lymphotropic HIV-1 $1_{\text {LAI }}$ or the macrophage-tropic HIV-1 $1_{\text {JR-FL }}$.

To clarify the nature of HIV-1 interaction with CAEC, we examined HIV-1 reverse transcription at 1 , 7 , and 10 days PI using primers for early (R/U5) and late (2LTR) steps designed according to the accepted model of retroviral reverse transcription. In CAEC exposed to HIV-1 $1_{\text {LAI }}, \mathrm{HIV}-1_{\text {JR-FL }}$ or HIV-1 $1_{89.6}$ at either low or high multiplicity, DNA PCR with R/U5 primers detected an early transcript, strong-stop DNA $R / U 5$. The level of transcription was increased in relation to the number of viral RNA copies in the inoculum but was generally very low (Fig. 3 ). In addition, in CAEC exposed to a high multiplicity of HIV- $\mathrm{I}_{\text {LAI }}(2000$ copies/cell), the pol transcript was also detected with the maximum level of expression 48 hr PI (Fig. 4). U3/R and 2 LTR transcripts were never detected after HIV-1 $1_{\text {LAI }}$ or HIV-1 $1_{J R-F L}$ infection of CAEC at any multiplicity (data not shown).

Time Course of HIV-1 Penetration Across CAEC and Lack of Inhibition by Chemokines

HIV- $1_{J R-F L}$ penetration across CAEC at $2 \mathrm{hr}$ PI was only 200 copies (the lower limit of sensitivity of the RNA assay is 50 copies) but at $4 \mathrm{hr}$ PI increased to 1000 copies and at $24 \mathrm{hr}$ PI to 3567 copies (an 

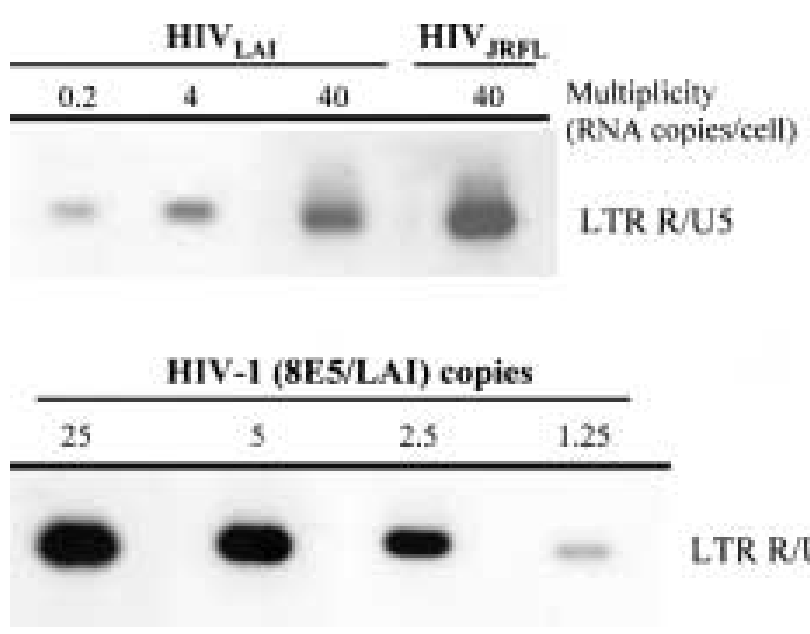

LTR R/US

Fig. 3. HIV-1 R/U5 reverse transcription in CAEC. Two hundred fifty thousand CAEC in a 6-well plate were infected with HIV-1 $1_{\text {LAI }}$ at the multiplicity of $0.2,4$, or 40 RNA copies per cell and HIV-1 $1_{J R-F L}$ at 40 copies per cell. The virus stocks were pretreated with $400 \mathrm{U} / \mathrm{mL}$ RNase-free DNase I, as described in Materials and Methods. PCR lysates were prepared 7 days PI and were subjected to PCR amplification using primers from the LTR/U5 region, the pol gene, or 2 LTR, and Southern blot hybridization with the appropriate $\mathrm{P}^{32}$-labeled probe. Serial dilutions of 8E5/LAI cells, containing one provirus per cell, were amplified with the same set of LTR/U5 primers as PCR standards. Reverse transcripts were detected at 7 days PI using only the LTR/U5 primers (data shown). No hybridization was obtained using the pol and 2LTR primers (data not shown).

increase of $1783 \%$ compared to the 2 -hr PI titer). The barrier showed a further breakdown at $48 \mathrm{hr}$ PI (Fig. 5). This experiment was repeated twice with similar results. Pretreatment of CAEC with AOP-

\section{Time After Infection}

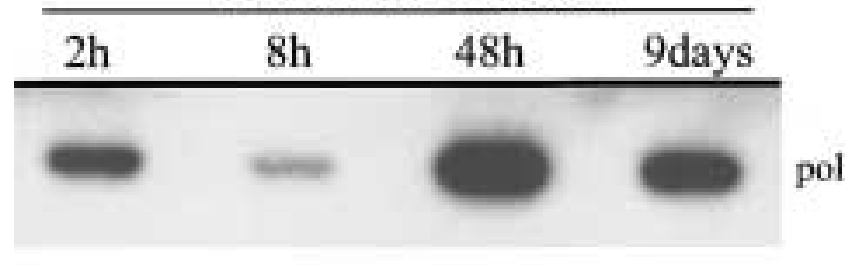

\section{HIV-1 (8E5/LAI) Copies}

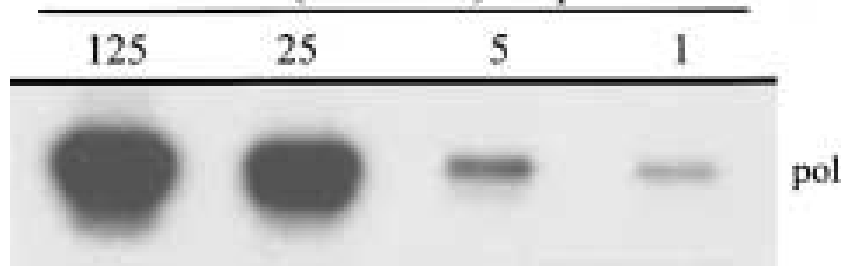

Fig. 4. HIV-1 pol reverse transcription in CAEC. Two hundred fifty thousand CAEC in a 6-well plate were infected with HIV- $1_{\text {LAI }}$ at the multiplicity of 2000 copies per cell. PCR lysates prepared at the indicated times PI were amplified using pol primers and were detected using the pol probe. The dilutions of 8E5/LAI cells and lysates of infected CEM cells were amplified with the same set of $\mathrm{pol}$ primers.

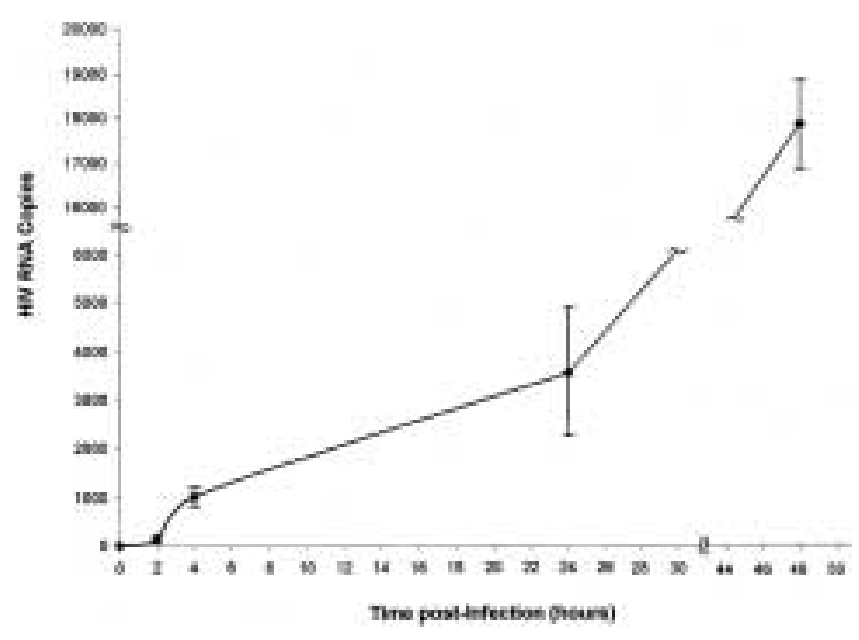

Fig. 5. Time course of HIV-1 penetration across CAEC. Triplicate CAEC models (prepared with CAEC in passage 6) were challenged with 1 million RNA copies of HIV- $1_{\text {JR-FL }}$ placed in the upper chamber. The inulin permeability coefficient baseline was determined in two replicate models as 0.000235 and $0.000256 \mathrm{~cm} / \mathrm{min}$. At indicated times, $100 \mu \mathrm{L}$ samples were removed from the lower chamber and RNA copy number was determined by the Amplicor assay.

RANTES or v-MIP II had no significant effect on virus penetration $24 \mathrm{hr}$ after infection (Table 1). In this experiment we removed the virus from the upper chamber $4 \mathrm{hr}$ PI, because we considered a possibility that the chemokines could block the receptors for only a short period of time. In two other experiments, the virus was not removed from the upper chamber and the results again showed no difference in virus penetration in the models treated or untreated with the chemokines (data not shown).

Table 1. Lack of inhibition by chemokines of HIV-1 JR-FL penetration across a CAEC barrier

\section{Pretreatment} of CAEC barrier

Control medium

Medium with AOP-RANTES (100 nM)

Medium with v-MIP II (100 nM)
HIV-1 penetration into the lower chamber (number of RNA copies) (duplicate titers)

CAEC barrier was treated with the indicated medium for $2 \mathrm{hr}$ prior to infection of the upper chamber with 1 million RNA copies of HIV- $1_{J R-F L}$. Virus-containing medium in the upper chamber was changed $4 \mathrm{hr}$ PI to the appropriate medium without virus. Twenty-four-hour PI samples were collected from the lower chamber and the concentration of HIV-1 RNA copies was determined by the Amplicor test. 

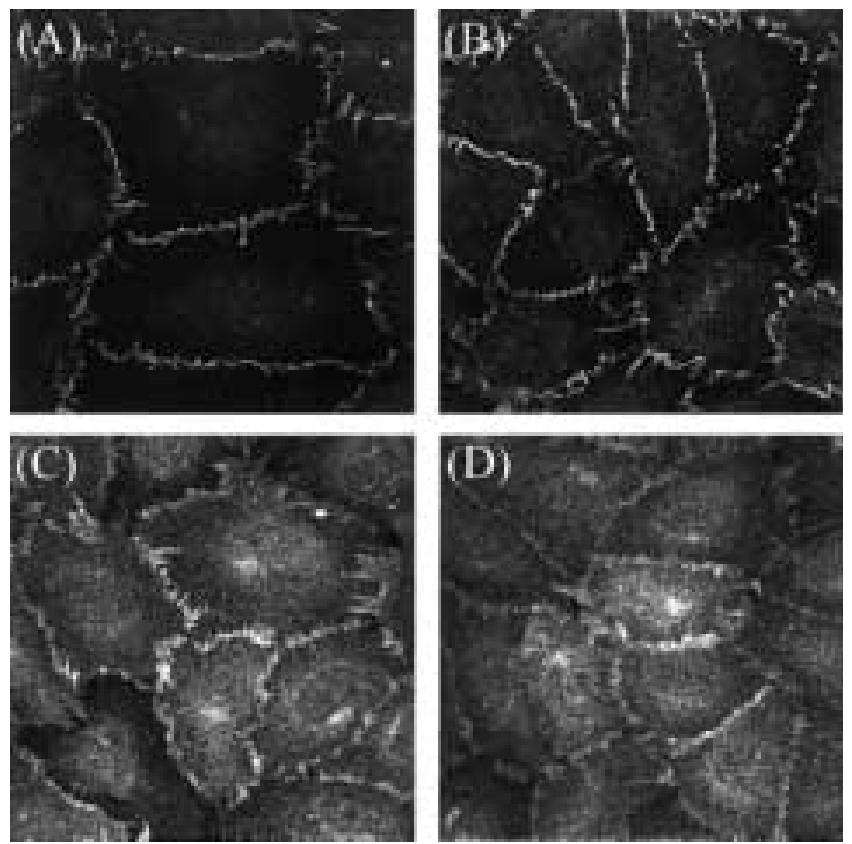

Fig. 6. Virus infection of CAEC does not alter tight junction proteins occludin and ZO-1. One hundred thousand CAECs were grown to confluence on glutaraldehyde-fixed, gelatin-coated 16-well chamber slides for 5 days. The cells were either infected with HIV- $1_{\mathrm{NL4}-3}$ at a multiplicity of 2500 RNA copies/cell for 4 days (A and C) or were sham infected (B and D). The cells were fixed for 20 min with $4 \%$ paraformaldehyde at room temperature and were stained by immunofluorescence using ZO-1 antibody (A and B) or occludin antibody (C and D). The images were obtained using Zeiss LSM 310 confocal laser microscope.

\section{Cell-Free HIV-1 Does Not Alter Paracellular Permeability}

The inulin permeability coefficient of untreated CAEC models was $0.000235-0.000256 \mathrm{~cm} / \mathrm{min}$, which is similar to that determined previously with Madin-Darby canine kidney cells, a tight epithelial cell monolayer (3). The inulin permeability was compared in CAEC models that were either cultured for $\mathbf{2 4} \mathrm{hr}$ in medium after sham infection or were infected with HIV-1 $1_{\text {LAI }}$ at a multiplicity of 400,000 RNA copies per cell and then cultured for $24 \mathrm{hr}$. The permeability coefficient of virus-infected models was not significantly changed by virus exposure. The average increase in the three replicate models treated with HIV-1 was $25 \%(p>.10)$.

\section{Microscopic and Submicroscopic Changes in Virus-Infected Cells}

Microscopic examination of virus-infected CAEC revealed no alterations of $\mathrm{TJ}$ as shown by confocal imaging of TJ stained with anti-ZO-1 or antioccludin (Fig. 6).

Transmission electron microscopy of CAEC exposed to a multiplicity of $1.5 \times 10^{6}$ RNA copies of HIV- $1_{\text {NL4-3 }}$ per cell revealed extensive cytoplasmic vacuolization developing 3-24 hr PI. The vacuoles had an average diameter of 100-500 nm. Virion-like

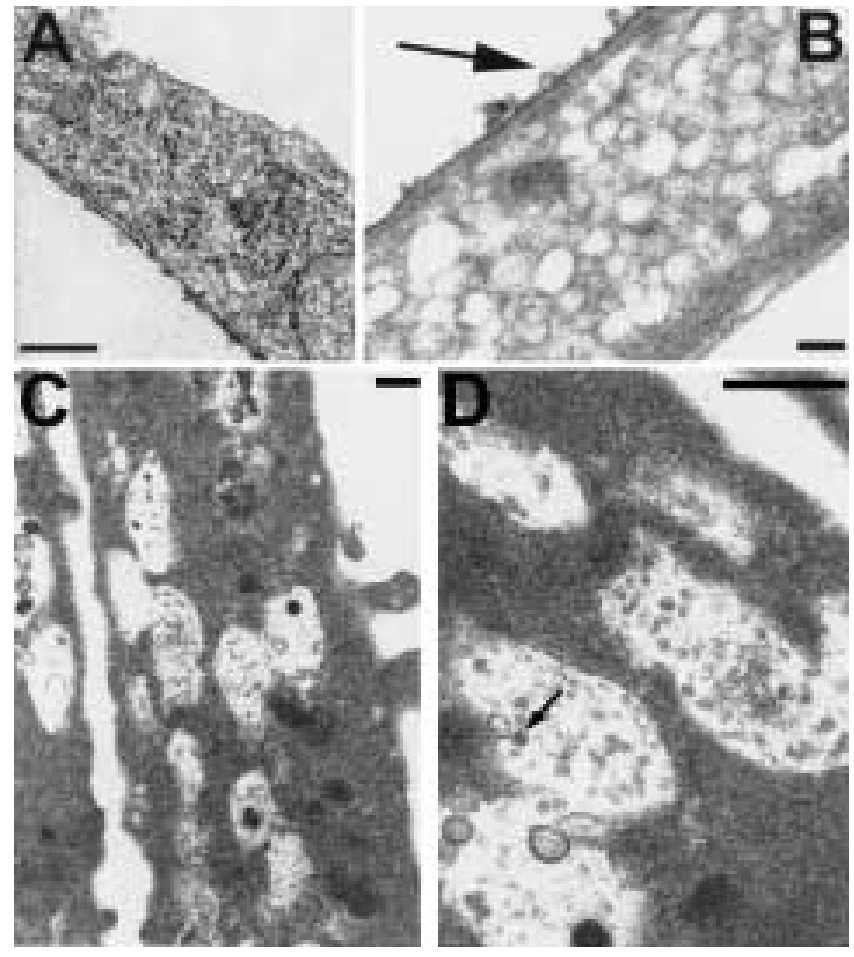

Fig. 7. HIV-1 exposure of CAEC or BMVEC induces cell vacuolization. Retroviral-like virions penetrate into the vacuoles. CAEC were either sham infected (A) or infected with HIV-1 at the multiplicity of $1.5 \times 10^{6}(\mathrm{~B})$. Note intense vacuolization of virus-exposed endothelia and virion-like particles (80-100 $\mathrm{nm}$ diameter) on the plasma membrane (arrow). BMVEC exposed to HIV-l at the same multiplicity also became vacuolated (C and $\mathrm{D})$ and retroviral particles with a dense core (the particle shown with an arrow has a diameter of approximately $100 \mathrm{~nm}$ ) were present in the vacuoles (C and D).

[Transmission electron microscopy; the bar indicates $1 \mu \mathrm{m}$ (A), $200 \mathrm{~nm}$ (B), $1 \mu \mathrm{m}$ (C), $1 \mu \mathrm{m}$ (D).]

particles (diameter $80 \mathrm{~nm}$ ) were observed in these vacuoles (Fig. 7A and B). This experiment was repeated in CAEC three times with similar results. Subsequently, BMVEC were exposed to HIV- $1_{\text {NL4-3 }}$ at approximately the same multiplicity. At a high magnification, the viral-like particles were visualized by electron microscopy in the vacuoles in BMVEC. The particles had a dense core and their diameter (100-120 nm) was consistent with the diameter of HIV-1 virions (Fig. 7C and D).

\section{Discussion}

The pathogenetic mechanisms of HIV-1 myocardiopathy are not understood, but appear to involve invasion of the myocardium by HIV-1. Our study provides strong evidence that HIV-1 may invade the myocardium by transcytosis across CAEC. Our CAEC were derived from coronary vessels and displayed factor VIII, ICAM-2, $\beta$-catenins, and occludin in the cytoplasm; and PECAM-1, $\beta$-catenins, ZO- 1 , and occludin in periplasmic distribution. Because these proteins are made in the 
endoplasmic reticulum and are transported through the cytoplasm, their staining (except ZO-1) was superimposed over the nucleus and the perinuclear Golgi apparatus. Viral invasion of the heart across CAEC had four special features.

First, productive infection of CAEC was ruled out by microscopic (transmission electron microscopy and immunofluorescence) and molecular studies. HIV-1 infection of CAEC was limited to reverse transcription of strong-stop DNA. This type of abortive infection differs from previously reported productive and nonproductive types of infection of other endothelia. For example, liver (26) and glomerular capillary (27) endothelia were permissive for productive infection, whereas brain microvessel endothelia were either permissive (16) or infected in a latent fashion (17).

Previous ex vivo studies of the AIDS brain suggested a possibility of productive infection of endothelia. For example, some microvessels showed HIV-1 antigen on immunostaining (28), and a combination of in situ DNA PCR, RT-initiated in situ PCR, and immunostaining suggested productive infection of endothelia (29). However, the presence of viral particles in endothelia taken into the cells by pinocytosis and a possibility of endogenous reverse transcription in the incoming virions (30) render the interpretation of these in vivo studies difficult.

Second, only a small fraction-at $24 \mathrm{hr}$ PI at most $6 \%$ of viral RNA copies-penetrated across the CAEC barrier, suggesting that endothelia are able to inactivate most of the infectious virus. Transmission electron microscopy demonstrated dramatic vacuolar remodeling of endothelia. Some virion-like particles were noted on the plasma membrane or inside the vacuoles of CAEC. A subsequent study of this phenomenon in BMVEC revealed extensive penetration of retroviral-like particles into the endothelial vacuoles, suggesting that HIV-1 can force its entry into the target organs, such as the heart and the brain.

Third, although multiple blocks to HIV-1 replication may exist, variable expression of HIV-1 coreceptors in different endothelia could be an important determinant of the efficiency of virus infection. The expression of the critical receptors CCR5, CXCR4, and CCR3 differs between brain and coronary endothelia in its density and subcellular distribution (25). Because the HIV-1 co-receptors CCR5 and CXCR4 are present on CAEC, we speculated that HIV-1 could use these receptors to engage and penetrate the endothelia in the vacuoles. However, vMIP-II and RANTES, which block virus entry through CCR3 and CCR5 (31), did not inhibit virus penetration, suggesting either that these receptors are not involved or that the chemokine antagonists used in this study do not prevent receptor recycling in endothelia, as they do in leukocytes (32).

Fourth, although virus penetration across endothelia could also occur through tight and adherens junctions, cell-free HIV-1 infection had no signifi- cant effect on transmembrane (occludin) and peripheral (ZO-1) TJ proteins, as shown by confocal microscopy and the ${ }^{14} \mathrm{C}$-inulin permeability assay. However, in vivo opening of $\mathrm{TJ}$ is mediated by inflammatory molecules, such as thrombin and histamine (33) or abused drugs, such as cocaine (4), which were not present in our cell culture system. A recent study noted that HIV-1 Tat increases endothelial permeability to fluorescein isothiocyanate labeled BSA (34). The dichotomy between the lack of HIV-1 effect on inulin permeability and the strong effect of Tat on albumin permeability may not be incongruous, because these target molecules are vastly different in size.

In conclusion, CAEC do not present an absolute barrier to cell-free HIV-1. During the primary infection associated with intensive plasma viremia, cellfree virus may open a transcytotic route, which could coexist with the paracellular route breached by cytokines and abused drugs.

\section{Acknowledgments}

This work was supported by DA10442, HL63639, and HL63065 to M.F., AI42557 to W.P., and HL42550 to A.R.B. We acknowledge the efforts of R. Offord and B. Dufour in synthesizing AOP-RANTES, and M. Witte, M. Weinand, and B. Krieger in providing BMVEC.

\section{References}

1. Barbaro G, Di Lorenzo G, Grisorio B, Barbarini G. (1998) Incidence of dilated cardiomyopathy and detection of HIV in myocardial cells of HIV-positive patients. Gruppo Italiano per lo Studio Cardiologico dei Pazienti Affetti da AIDS [see comments]. N. Engl. J. Med. 339: 1093-1099.

2. Barbaro G, Di Lorenzo G, Grisorio B, Barbarini G. (1998) Cardiac involvement in the acquired immunodeficiency syndrome: a multicenter clinical-pathological study. Gruppo Italiano per lo Studio Cardiologico dei pazienti affetti da AIDS Investigators. AIDS Res. Hum. Retroviruses 14: 1071-1077.

3. Fiala M, Looney DJ, Stins M, et al. (1997) TNF-alpha opens a paracellular route for HIV-1 invasion across the blood-brain barrier. Mol. Med. 3: 553-564.

4. Zhang L, Looney D, Taub D, et al. (1998) Cocaine opens the blood-brain barrier to HIV-1 invasion. J. Neurovirol. 4: 619-626.

5. Brightman MW, Ishihara S, Chang L. (1995) Penetration of solutes, viruses, and cells across the blood-brain barrier. Curr. Top. Microbiol. Immunol. 202: 63-78.

6. Marsh M, Helenius A. (1989) Virus entry into animal cells. Adv. Virus Res. 36: 107-151.

7. Anderson HA, Chen Y, Norkin LC. (1996) Bound simian virus 40 translocates to caveolin-enriched membrane domains, and its entry is inhibited by drugs that selectively disrupt caveolae. Mol. Biol. Cell 7: 1825-1834.

8. Koukouritaki SB, Vardaki EA, Papakonstanti EA, et al. (1999) TNF-alpha induces actin cytoskeleton reorganization in glomerular epithelial cells involving tyrosine phosphorylation of paxillin and focal adhesion kinase. Mol. Med. 5: 382-392.

9. Molony L, Armstrong L. (1991) Cytoskeletal reorganizations in human umbilical vein endothelial cells as a result of cytokine exposure. Exp. Cell Res. 196: 40-48.

10. Wojciak-Stothard B, Entwistle A, Garg R, Ridley AJ (1998) Regulation of TNF-alpha-induced reorganization of the actin 
cytoskeleton and cell-cell junctions by Rho, Rac, and Cdc42 in human endothelial cells. J. Cell Physiol. 176: 150-165.

11. Ali MH, Schlidt SA, Chandel NS, et al. (1999) Endothelial permeability and IL-6 production during hypoxia: role of ROS in signal transduction. Am. J. Physiol. 277: L1057-1065.

12. Feng D, Nagy JA, Pyne K, et al. (1999) Pathways of macromolecular extravasation across microvascular endothelium in response to VPF/VEGF and other vasoactive mediators. Microcirculation 6: 23-44.

13. Mullberg J, Geib T, Jostock T, et al. (2000) IL-6 receptor independent stimulation of human gpl30 by viral IL-6. J. Immunol. 164: 4672-4677.

14. Peluso R, Haase A, Stowring L, Edwards M, Ventura P. (1985) A Trojan Horse mechanism for the spread of visna virus in monocytes. Virology 147: 231-236.

15. Gan X, Zhang L, Taub D, et al. (1999) Cocaine enhances endothelial adhesion molecules and leukocyte migration. Clin. Immunol. 91: 68-76.

16. Moses AV, Bloom FE, Pauza CD, Nelson JA (1993) Human immunodeficiency virus infection of human brain capillary endothelial cells occurs via a CD4/galactosylceramideindependent mechanism. Proc. Natl Acad. Sci. USA 90: 10474-10478.

17. Poland SD, Rice GP, Dekaban GA. (1995) HIV-1 infection of human brain-derived microvascular endothelial cells in vitro. J. Acquir. Immune Defic. Syndr. Hum. Retrovirol. 8: 437-445.

18. Conaldi P, Serra C, Dolei A, et al. (1995) Productive HIV-1 infection of human vascular endothelial cells requires cell proliferation and is stimulated by combined treatment with interleukin-1 beta plus tumor necrosis factor-alpha. J. Med. Virol. 47: 355-363.

19. Ades EW, Comans TW, Nicholson JK, Browning SW. (1993) Lack of evidence that human immunodeficiency virus can infect human endothelial cells in vitro [letter]. J. Acquir. Immune Defic. Syndr. 6: 104.

20. Folks TM, Powell D, Lightfoote M, et al. (1986) Biological and biochemical characterization of a cloned Leu-3- cell surviving infection with the acquired immune deficiency syndrome retrovirus. J. Exp. Med. 164: 280-290.

21. Simmons G, Clapham PR, Picard L, et al. (1997) Potent inhibition of HIV-1 infectivity in macrophages and lymphocytes by a novel CCR5 antagonist. Science 276: 276-279.

22. Popik W, Pitha PM. (1996) Binding of human immunodeficiency virus type 1 to CD4 induces association of Lck and
Raf- 1 and activates Raf- 1 by a Ras-independent pathway. Mol. Cell Biol. 16: 6532-6541.

23. Burns AR, Walker DC, Brown ES, et al. (1997) Neutrophil transendothelial migration is independent of tight junctions and occurs preferentially at tricellular corners. J. Immunol. 159: 2893-2903.

24. Schmidtmayerova H, Alfano $M$, Nuovo G, Bukrinsky M (1998) Human immunodeficiency virus type I T-lymphotropic strains enter macrophagesvia a CD4- and CXCR4mediated pathway: replication is restricted at a postentry level. J. Virol. 72: 4633-4642.

25. Berger O. (1999) Chemokine receptors on coronary and brain endothelia. Mol. Med. 5: 795-805.

26. Steffan AM, Lafon ME, Gendrault JL, et al. (1992) Primary cultures of endothelial cells from the human liver sinusoid are permissive for human immunodeficiency virus type 1 . Proc. Natl Acad. Sci. USA 89: 1582-1586.

27. Green DF, Resnick L, Bourgoignie JJ. (1992) HIV infects glomerular endothelial and mesangial but not epithelial cells in vitro. Kidney Int. 41: 956-960.

28. Wiley CA, Schrier RD, Nelson JA, Lampert PW, Oldstone MB. (1986) Cellular localization of human immunodeficiency virus infection within the brains of acquired immune deficiency syndrome patients. Proc. Natl. Acad. Sci USA 83: 7089-7093.

29. Bagasra O, Lavi E, Bobroski L, et al. (1996) Cellular reservoirs of HIV-1 in the central nervous system of infected individuals: identification by the combination of in situ polymerase chain reaction and immunohistochemistry. AIDS 10: 573-585.

30. Zhang H, Dornadula G, Pomerantz RJ. (1998) Natural endogenous reverse transcription of HIV type 1. AIDS Res. Hum. Retroviruses 14 Suppl 1: S93-95.

31. Kledal TN, Rosenkilde MM, Coulin F, et al. (1997) A broadspectrum chemokine antagonist encoded by Kaposi's sarcomaassociated herpesvirus. Science 277: 1656-1659.

32. Elsner J, Mack M, Bruhl H, et al. (2000) Differential activation of CC chemokine receptors by AOP-RANTES. J. Biol. Chem. 275: 7787-7794.

33. Dejana E, Del Maschio A. (1995) Molecular organization and functional regulation of cell to cell junctions in the endothelium. Thromb. Haemost. 74: 309-312.

34. Oshima T, Flores SC, Vaitaitis G, et al. (2000) HIV-1 Tat increases endothelial solute permeability through tyrosine kinase and mitogen-activated protein kinase-dependent pathways. AIDS 14: 475-482. 